\title{
TOP CITED PAPERS IN INTERNATIONAL PSYCHOGERIATRICS: 4. EFFECTS OF INDIVIDUALIZED VS. CLASSICAL "RELAXATION" MUSIC ON THE FREQUENCY OF AGITATION IN ELDERLY PERSONS WITH ALZHEIMER'S DISEASE AND RELATED DISORDERS
}

\section{Reflection}

While working as a staff development coordinator in a long-term care facility during the mid to late 1980 s I witnessed agitation in persons with dementia (PWD) and the negative effects that these behaviors had on both the caregiver and the care recipient. Published research findings validated the widespread prevalence of this problem. Management strategies at the time focused primarily on chemical and physical restraints which, in and of themselves, were fraught with adverse effects. Shortly thereafter I enrolled in graduate school where I focused my efforts on exploring this problem with a clear understanding that there was a need for alternative interventions that were relatively inexpensive and could be readily and easily implemented by trained staff. These efforts eventually led to the development of individualized music as an intervention for the management of agitation in PWD. Individualized music is defined as music that has been integrated into the person's life and is based on personal preference (Gerdner, 1992). Extensive clinical experience along with findings from a pilot study (Gerdner, 1992) served as the foundation for the development of intervention guidelines (Gerdner, 1996) and a midrange theory of individualized music intervention for agitation (IMIA) (Gerdner, 1997). The publication of the original pilot study (Gerdner and Swanson, 1993) generated a considerable amount of interest resulting in further efforts toward testing the effects of individualized music for the management of agitation in PWD (Casby and Holm, 1994; Cohen-Mansfield and Werner, 1997; Devereaux, 1997; Thomas et al., 1997; Clark et al., 1998). The strengths and limitations of these studies were used to design a more rigorous methodology using a larger sample for the purpose of testing the propositions of IMIA and the effects of individualized music when compared to classical "relaxation" music on the frequency of agitation in PWD. The findings of this study were published in International Psychogeriatrics (Gerdner, 2000). As of January 2009, this article has been cited in 91 scholarly publications and was the impetus for additional studies conducted in the U.S.A., Sweden, Japan and Taiwan. These efforts have resulted in an

First published online 15 April 2009. expanding body of research to support the use of this intervention for the management of agitation (Ragneskog et al., 2001; Janelli et al., 2002; Suzuki et al. 2004; Sung et al., 2006; 2008; Park, 2008).

This paper begins by providing a brief summary of the underlying theory for the use of individualized music and advances to a description of the Assessment of Personal Music Preference and the evidence-based guidelines for its implementation. A summary of key research efforts to evaluate the use of individualized music in PWD (conducted since 2000 ) is provided. Next, characteristics are explored that may have led to the growing interest in individualized music as an alternative intervention for the management of agitation in PWD. The paper concludes with recommendations for future research.

\section{Mid-range theory}

The mid-range theory of individualized music intervention for agitation (IMIA) (Gerdner, 1997) serves as the theoretical basis for this intervention. Elements of the mid-range theory include: cognitive impairment, progressively lowered stress threshold, agitation, and individualized music. Briefly summarized, cognitive impairment results in a decreased ability to receive and process stimuli, resulting in a progressive decline in the person's stress threshold (Hall and Buckwalter, 1987). Dysfunctional behaviors (i.e. agitation) occur when the stress threshold is exceeded (Hall and Buckwalter, 1987). Music may be used as a means of communicating with this population even in the advanced stages of dementia when the person is unable to understand verbal language and has a decreased ability to interpret environmental stimuli. It is theorized that the presentation of individualized music (carefully selected music, based on personal preference) will provide an opportunity to stimulate remote memory. This changes the focus of attention and provides an interpretable stimulus, overriding stimuli in the environment that is meaningless or confusing. The elicitation of memories associated with positive feelings will have a soothing effect on the person with dementia, which in turn will prevent or alleviate agitation (Gerdner, 1997).

\section{Assessment of Personal Music Preference}

The Assessment of Personal Music Preference (APMP) was developed by Gerdner, Hartsock 
and Buckwalter in 2000 and is included in the evidence-based protocol (Gerdner, 2007a). APMP was developed to assist in the selection of individualized music. Questions are used to explore the meaning of music in the person's life and identify preferences in song titles. Because musical selections are often closely aligned with specific performers this also becomes an important part of the assessment process.

When cognitive impairment prevents the person from identifying or expressing their own personal preference an alternate version is available for completion by a close family member. The alternative version of APMP has been successfully used by family members of residents living in long-term care facilities (Gerdner, 2005) and those who care for an elder at home (Park, 2008). Both versions of the APMP are contained in the evidencebased guideline described in the next section.

\section{Evidence-based guideline}

An evidence-based guideline for the implementation of individualized music by professional health care providers has been developed and refined over time (Gerdner, 1996, updated: 1999, 2001, 2007a) based on the expanding body of evidence. This guideline is accompanied by an evidence grade schema. The schema is used to assign a specific grade based on the strength and type of evidence for each recommendation within the guideline. A simplified, more concise version has been created for consumers (i.e. family caregivers) (Gerdner, 2001, updated: 2007b).

The guideline describes the assessment criteria for use of pre-recorded music for the purpose of alleviating agitation in persons with dementia. The guideline also includes a recommendation for assessing the temporal patterning of agitation so that the timing of the intervention can be tailored to maximize the effects and benefits of the intervention.

\section{Advancing the science}

Japanese researchers (Suzuki et al. 2004) expanded the evaluation of preferred music in PWD by adding functional and biophysiological measures as well as behavioral outcome measures. The study included 10 subjects with dementia who received individualized or preferred music twice per week for 8 weeks. During the corresponding period, 13 subjects participated in a comparison intervention (games, drawing, pasting pictures). Analysis, comparing baseline to one-week post intervention scores, found that subjects in the experimental group had a statistically significant improvement in the "language" subscale of the Mini-mental
State Examination and a statistically significant reduction in "irritability" as measured by the Multidimensional Observational Scale. In addition, there was a statistically significant reduction in salivary chomogranin A ( CgA) following session 16. The authors concluded, that "the changes in CgA levels supported Gerdner's mid range theory" (p. 17). No significant findings occurred in the control group across outcome measures.

\section{Translating research into practice}

Given its efficacy when implemented by research staff, it is important to evaluate the effectiveness of individualized music when implemented by trained staff and family members. As a first effort, Gerdner (2005) conducted a pilot study using both quantitative and qualitative measures. Following appropriate training, staff and family implemented individualized music to eight PWD living in a longterm care facility. The intervention was implemented over a 4-week period. Individualized music was played daily for 30 minutes at a prescribed time (prior to the estimated "peak level" of agitation). The mean rate of compliance was $86.3 \%$. In addition, staff administered music on an as needed basis (when the PWD first began exhibiting signs of agitation). Agitation was measured using a modified version of the Cohen-Mansfield Agitation Inventory. A statistically significant reduction in agitation was found during the immediate presentation of music with an overall reduction in agitation being found on day shift during weeks 1-8 and on evening shift during weeks $5-8$. Staff and family interviews provided convergent validity to quantitative findings. In addition, staff and family reported that individualized music provided a catalyst for meaningful interaction between the person with dementia and others.

Researchers in Taiwan also evaluated the implementation of individualized music when implemented by trained staff in a long-term care facility. Findings were published in two separate articles. In the first article (Sung et al., 2008) the researchers focused on knowledge and adherence to the evidence-based guideline for individualized music when implemented by 17 nursing staff working in a long-term care facility in Taiwan. Initial training included an interactive educational program. Ongoing reminders, a local opinion leader, and an audit checklist were used to facilitate and monitor continued adherence to the intervention protocol. Analysis used to compare pre- and post-test scores found a statistically significant improvement $(p<0.001)$ in knowledge of the intervention following the training session with a mean compliance of $72 \%$. 
The second article (Sung et al., 2006) focused on the resident's response to individualized music as measured by the Cohen-Mansfield Agitation Inventory (CMAI). The sample included an experimental group $(\mathrm{n}=32)$ that received individualized music for 30 minutes, twice per week over 6 weeks. The control group $(n=25)$ received usual care without music. Findings showed that the experimental group had a statistically significant reduction in overall agitation $(\mathrm{t}=-2.19, \mathrm{p}<0.05)$ and physically non-aggressive behaviors $(\mathrm{t}=-3.75$, $\mathrm{p}<0.0001$ ) compared to the comparison group.

In 2008, Park trained 20 in-home family caregivers on the use of the evidence-based guideline for individualized music. Outcome measures included the modified Cohen-Mansfield Agitation Inventory and the Modified Pain Assessment in the Demented Elderly. A quasi-experimental design was used in which individualized music was implemented two times per week for two weeks. Statistical analysis found a significant reduction in agitation and pain during the intervention period compared to baseline and post-intervention periods.

\section{Dissemination}

Continuing efforts are underway to promote the use of individualized music by clinicians. For example, to educate nurses in the assessment, implementation, and evaluation of individualized music, an interactive educational module has been developed. Learners are then given the opportunity to apply their knowledge to a case study that focuses on an Italian-American woman diagnosed with dementia (Gerdner, 2004). The training module is available through the Sigma Theta Tau Online Continuing Education Program and has recently been updated (Gerdner, 2008).

The New York State Department of Health has developed a series of multidisciplinary online training modules referred to as the Electronic Dementia Guide for Excellence. One of the modules specifically focuses on individualized music (Gerdner, 2006). The module provides specific guidelines for the development of a 45-minute in-service training session, including learning objectives and a PowerPoint presentation. A sample case study is provided to assist the learner in the application of

\section{Commentary}

The paper by Linda A. Gerdner published in International Psychogeriatrics (Gerdner, 2000) won third place in the 1999 International Psychogeriatric Association (IPA)/Bayer Research Awards in this knowledge for the assessment, implementation, and evaluation of individualized music.

\section{Popularity}

Individualized music was developed as an alternative intervention for the management of agitation in PWD. The use of pre-recorded music does not require special musical abilities and can therefore be implemented by trained staff and family caregivers. The positive response of the recipient to the music has been identified as a facilitator for its continued use by staff and family (Gerdner, 2005). The intervention promotes a humanistic, individualized approach to care. The process of assessing and implementing music has been shown to promote a collaborative working relationship between staff and family (Gerdner, 2005).

\section{Research implications}

As research evolves, methods and outcome measures are beginning to capture a more global response to individualized music. There is need to build and expand the work of Suzuki and colleagues (2004) through the use of biophysiological and functional outcome measures. In addition, anecdotal data and findings from open-ended interviews indicate a positive behavioral response while listening to preferred music that "illuminates personhood" of the listener (Gerdner, 1992; 2000; 2005). However, the recipient's positive response needs to be measured through more quantifiable outcome measures. There is also need to design large feasibility studies that evaluate the cost effectiveness of individualized music when implemented by staff in facilities such as long-term care. Although great strides have been made toward building a body of evidence for the use of individualized music in persons with dementia many exciting opportunities await. Individualized music - let the beat go on ...

\section{LINDA A. GERDNER \\ Consulting Assistant Professor, Stanford Geriatric \\ Education Center, Center for Education in Family and \\ Community Medicine, Stanford University School of \\ Medicine, California, U.S.A. \\ Email: lgerdner@gmail.com}

Psychogeriatrics. In doing so it follows the tradition of many of the papers taking first, second or third place in this award scheme in yielding high citation scores over subsequent years (McKeith and Ames, 2009) - even by 2006 it had been cited 45 times in peer-reviewed journals. 
One does not need to search far to understand why Linda Gerdner's paper has enjoyed this level of interest. It is an excellent example of a feasible and successful non-pharmacological intervention for behavioral and psychological symptoms of dementia (BPSD). Based on a theory of why the intervention should help, it manages the difficult step of translating this theory into the practice of dementia care in long-term care facilities by adopting an inexpensive methodology that can be adopted by regular staff after adequate training and does not require extensive supervision by staff. The study acknowledges that changing the culture of the management of BPSD in institutions can only succeed if the intervention is incorporated into the framework of that institution and works within the limitations of the environment, rather than expecting unrealistic modifications. In addition, by using deliberately generous inclusion criteria with a wide range of severity and causes of cognitive impairment, the intervention - in this case individualized music - has a better chance of being adopted after completion of the research project, since it does not require complicated assessments of the residents. This has also encouraged other research teams to try the intervention in different settings. This "user-friendly" approach has been combined with a rigorous statistical methodology to produce high quality research results. Furthermore, this approach has been very important in convincing clinicians, service administrators and even family members that the use of non-pharmacological interventions should be first-line management for BPSD.

Agitation is one of the most challenging manifestations of BPSD with which family and professional carers must cope, and patients with agitation are at risk of injury and to being exposed to potentially dangerous pharmacological management (Spira and Edelstein, 2006). To most of us, listening to our own musical preferences is a pleasurable experience because we tend to associate such music with positive events earlier in our lives. It can therefore offer access to memory when cognitive impairment has blocked many other such openings (faceto-face verbal communication, reading, writing, travelling, etc.). This positive connection between music and memory seems, for many patients, to last well into the advanced stages of cognitive impairment (Gerdner, 2000). The unique approach of Gerdner's "mid-range theory of individualized music intervention for agitation" acknowledges the importance of focusing on the patient as an individual, especially in an environment where individual preferences are often difficult to meet. Her work highlights the challenge for researchers and clinicians involved in the care of patients with cognitive impairment never to lose sight of the essential questions: who is the person suffering from BPSD and what life has he or she lived?

\section{Nicola T. LaUtenschlageR}

Deputy Editor of International Psychogeriatrics, Melbourne, Australia

Email: nicolatl@unimelb.edu.au

\section{References}

Casby, J. A. and Holm, M. B. (1994). The effect of music on repetitive disruptive vocalizations of persons with dementia. American fournal of Occupational Therapy, 48, 883-889.

Clark, M. E., Lipe, A. W. and Bilbrey, M. (1998). Use of music to decrease aggressive behaviors in people with dementia. Fournal of Gerontological Nursing, 24, 10-17.

Cohen-Mansfield, J. and Werner, P. (1997). Management of verbally disruptive behaviors in nursing home residents. fournal of Gerontology. Series A, Biological Sciences and Medical Sciences, 52A, M369-377.

Devereaux, M. A. (1997). The effects of individualized music on cognitively impaired nursing home residents exhibiting agitation. Unpublished Masters thesis, College of St. Catharine, St. Paul, Minnesota.

Gerdner, L. A. (1992). The effects of individualized music on elderly clients who are confused and agitated. Unpublished Masters thesis, University of Iowa

Gerdner, L. A. (1996, updated 1999, 2001). Evidence-based protocol: individualized music intervention. In M. Titler (series ed.) Series on Evidence-Based Practice for Older Adults. Iowa City, Iowa: The University of Iowa College of Nursing Gerontological Nursing Interventions Research Center, Research Dissemination Core.

Gerdner, L. A. (1997). An individualized music intervention for agitation. Fournal of the American Psychiatric Nurses Association, 3, 177-184.

Gerdner, L. A. (2000). Effects of individualized vs. classical "relaxation" music on the frequency of agitation in elderly persons with Alzheimer's disease and related disorders. International Psychogeriatrics, 12, 49-65.

Gerdner, L. A. (2001). Consumer version of evidence-based protocol: individualized music. In M. Titler (series ed.) Series on Evidence-Based Practice for Older Adults. Iowa City, Iowa: The University of Iowa College of Nursing Gerontological Nursing Interventions Research Center, Research Dissemination Core.

Gerdner, L. A. (2004). That's Amore! Reducing Agitation in an Italian-American Elder with Dementia through Individualized Music [Case Study SC0005. 1.1 CEU]. Nursing Knowledge International. Sigma Theta Tau International Online Continuing Education Program. http://www. nursingsociety.org/education/SC0005/sc0005_index.html

Gerdner, L. A. (2005). Use of individualized music by trained staff and family: translating research into practice. Fournal of Gerontological Nursing, 31, 22-30.

Gerdner, L. A. (2006). Intervention: Individualized music. In J. Ronch (ed.), The Electronic Dementia Guide for Excellence 
(EDGE) Project. Funded by the New York State

Department of Health. Available at: http://www.health.state. ny.us/diseases/conditions/dementia/edge/interventions/ indiv_music/index.htm

Gerdner, L. A. (2007a). Evidence-based guidelines: individualized music for elders with dementia. In: M. G. Titler (series ed.), Series on Evidence-Based Practice for Older Adults. Iowa City, Iowa: The University of Iowa College of Nursing Gerontological Nursing Interventions Research Center, Research Translation and Dissemination Core. http://www.guideline.gov/summary/summary.aspx?ss= 15anddoc_id $=10777$ andnbr $=5605$

Gerdner, L. A. (2007b). Consumer version of evidence-based guidelines: individualized music for elders with dementia. In M. Titler (series ed.) Series on Evidence-Based Practice for Older Adults. Iowa City, Iowa: The University of Iowa College of Nursing Gerontological Nursing Interventions Research Center, Research Dissemination Core.

Gerdner, L. A. (2008). That's Amore! Reducing Agitation in an Italian-American Elder with Dementia through Individualized Music, Sigma Theta Tau International Honor Society of Nursing/The John A. Hartford Foundation Online Continuing Education Program at http://www. nursingknowledge.org/stti_ce/GNLA001/gnla001_index. html.

Gerdner, L. A. and Swanson, E. A. (1993). Effects of individualized music on elderly patients who are confused and agitated. Archives of Psychiatric Nursing, 7, 282-291.

Hall, G. R. and Buckwalter, K. C. (1987). Progressively lowered stress threshold: a conceptual model for care of adults with Alzheimer's disease. Archives of Psychiatric Nursing, 1, 399-406.
Janelli, L., Kanski, G. and Wu, Y. (2002). Individualized music: a different approach to the restraint use. Rehabilitation Nursing, 27, 221-226.

McKeith, I. and Ames, D. (2009) Top cited papers in International Psychogeriatrics 1: Long-term use of rivastigmine in patients with dementia with Lewy bodies: an open-label trial. International Psychogeriatrics, 21, 5-6.

Park, H. (2008). The effect of individualized music on agitation in patients with dementia who live at home. Unpublished $\mathrm{PhD}$ dissertation. University of Iowa; Iowa City, Iowa.

Ragneskog, H., Asplund, K., Kihlgren, M. and Norberg, A. (2001). Individualized music played for agitated patients with dementia: analysis of video-recorded sessions. International Fournal of Nursing Practice, 7, 146-155.

Spira, A. P. and Edelstein, B. A. (2006) Behavioral interventions for agitation in older adults with dementia: an evaluative review. International Psychogeriatrics, 18, 195-225.

Sung, H., Chang, A. M. and Abbey, J. (2006). The effects of preferred music on agitation of older people with dementia in Taiwan. International fournal of Geriatric Psychiatry, 21, 999-1000.

Sung, H-C., Chang, A. M. and Abbey, J. (2008). An implementation programme to improve nursing home staff's knowledge of and adherence to an individualized music protocol. Fournal of Clinical Nursing, 17, 2573-2579.

Suzuki, M. et al. (2004). Behavioral and endocrinoligcal evaluation of music therapy for elderly patients with dementia. Nursing and Health Sciences, 6, 11-18.

Thomas, D. W., Heitman, R. J., and Alexander, T. (1997). The effects of music on bathing cooperation for residents with dementia. Fournal of Music Therapy, 34, 246-259. 